\title{
Solitomab, an EpCAM/CD3 bispecific antibody construct (BiTE®), is highly active against primary uterine serous papillary carcinoma cell lines in vitro
}

\author{
Stefania Bellone ${ }^{1}$, Jonathan Black ${ }^{1}$, Diana P. English ${ }^{1}$, Carlton L. Schwab ${ }^{1}$, Salvatore \\ Lopez $^{1}$, Emiliano Cocco ${ }^{1}$, Elena Bonazzoli ${ }^{1}$, Federica Predolini ${ }^{1}$, Francesca Ferrari ${ }^{1}$, Elena \\ Ratner ${ }^{1}$, Dan-Arin Silasi ${ }^{1}$, Masoud Azodi ${ }^{1}$, Peter E. Schwartz ${ }^{1}$, and Alessandro D. Santin ${ }^{1}$ \\ ${ }^{1}$ Department of Obstetrics, Gynecology \& Reproductive Sciences, Yale University School of \\ Medicine, New Haven, CT
}

\section{Abstract}

Background-Uterine serous carcinoma (USC) is an aggressive form of endometrial cancer which carries an extremely poor prognosis. Solitomab is a novel bispecific single-chain antibody construct which targets epithelial-cell-adhesion-molecule (EpCAM) on tumor cells and also contains a CD3 binding region. We evaluated the expression levels of EpCAM and the in vitro activity of solitomab against primary USC cell lines in vitro and ex-vivo in the ascites of USC patients.

\begin{abstract}
Objectives-To determine the frequency of expression of EpCAM on uterine serous carcinoma cell lines as well as the ability of solitomab to modulate immune responses (T-cell proliferation, activation, cytokine production, and tumor killing) to tumor cells when it is combined with lymphocytes and EpCAM positive cell lines or EpCAM positive acitic fluid in vitro.
\end{abstract}

\begin{abstract}
Study Design-EpCAM expression was evaluated by flow cytometry in a total of 14 primary USC cell lines. Sensitivity to solitomab-dependent-cellular-cytotoxicity (ADCC) was tested against a panel of primary USC cell lines expressing different levels of EpCAM in standard $4 \mathrm{~h}{ }^{51} \mathrm{Cr}$ release-assays. The proliferative activity, activation, cytokine secretion (i.e., Type I vs Type II) and cytotoxicity of solitomab in autologous tumor-associated-T cells (TAL) in the ascitic fluid of USC patients was also evaluated by CFSE and flow-cytometry assays. Differences in EpCAM expression, ADCC levels were analyzed using upaired $t$ test. T-cell activation marker increase and cytokine release were analyzed by paired $t$ test.
\end{abstract}

\begin{abstract}
Results-Surface expression of EpCAM was found in 85.7\% (12 out of 14) of the USC cell lines tested by flow cytometry. EpCAM positive cell lines were found resistant to NK or T-cellmediated killing after exposure to peripheral blood lymphocytes (PBL) in 4-hour chromium-
\end{abstract}

Corresponding Author: Alessandro D. Santin MD, Address: 333 Cedar Street, LSOG 305, PO Box 208063, New Haven, CT 06520-8063, USA, Telephone: 203-737-4450; Fax: 203-737-4339 alessandro.santin@yale.edu.

Publisher's Disclaimer: This is a PDF file of an unedited manuscript that has been accepted for publication. As a service to our customers we are providing this early version of the manuscript. The manuscript will undergo copyediting, typesetting, and review of the resulting proof before it is published in its final citable form. Please note that during the production process errors may be discovered which could affect the content, and all legal disclaimers that apply to the journal pertain.

CONFLICT OF INTEREST STATEMENT

The authors declare no conflict of interest or previous publication and fulfill all conditions required for authorship. 
release assays (mean killing \pm SEM, $2.7 \pm 3.1 \%$ after incubation of EpCAM positive cell lines with control BiTE®). In contrast, after incubation with solitomab, EpCAM positive USC cells became highly sensitive to $\mathrm{T}$ cell cytotoxicity (mean killing $\pm \mathrm{SEM}$ of $25.7 \pm 4.5 \%$; $\mathrm{P}<0.0001$ ) by PBL. Ex vivo incubation of autologous tumor associated lymphocytes (TAL) with EpCAM expressing malignant cells in ascites with solitomab, resulted in a significant increase in T-cell proliferation in both CD4+ and CD8+ T cells, increase in T-cell activation markers (i.e., CD25 and HLA-DR), and a reduction in number of viable USC cells in ascites $(\mathrm{P}<0.001)$.

Conclusions-Solitomab induces robust immunologic responses in vitro resulting in increased T-cell activation, proliferation, production of cytokines, and direct killing of tumor cells. These finding suggest that solitomab may represent a novel, potentially effective agent for treatment of recurrent/metastatic and/or chemo-resistant USC overexpressing EpCAM.

\section{Keywords}

EpCAM; CD3; T-lymphocyte; Bispecific antibody; Uterine serous cancer

\section{INTRODUCTION}

Cancer of the uterine corpus is the most prevalent gynecologic tumor in women, with an estimated 54,870 cases and 10,170 deaths in the United States in 2015². The majority of cancers of the uterus are early stage, low grade, and endometrioid tumors (i.e. Type I). These neoplasms are frequently diagnosed in younger women, are associated with a history of hyperestrogenism as the main risk factor, and typically have a favorable prognosis with appropriate therapy. In contrast, Type II endometrial cancers are poorly differentiated tumors, often with serous papillary (USC) or clear cell histology. USC accounts for a disproportionately large number of endometrial cancer deaths despite the fact that it is only found in $10 \%$ of women with newly diagnosed endometrial cancer. ${ }^{2-5}$.

With the goal of identifying novel diagnostic and therapeutic molecular markers against this deadly variant of endometrial cancer our group has recently evaluated the genetic landscape and gene expression profiling of a large number of uterine serous carcinomas ${ }^{6-8}$. This study demonstrated epithelial cell adhesion molecule (EpCAM, also known as TROP-1 or TACSTD1), a calcium-independent hemophilic cell adhesion molecule of 39 to $42 \mathrm{kd}$, as one of the top differentially expressed genes in $\mathrm{USC}^{9-10}$. The EpCAM protein consists of an extracellular domain with 2 epidermal growth factor-like repeats, as well as a transmembrane domain, and a short cytoplasmic domain of 26 amino acids. The physiologic function of EpCAM is to promote cell adhesion through low level expression on the basolateral and intercellular surface of simple, pseudo-stratified, and transitional epithelia, including most epithelial tissues in the female genital tract ${ }^{9}$. EpCAM is a multifunctional protein and takes part in mediating cell signaling, cell migration, proliferation, and differentiation. Importantly, because of its relatively low expression in normal tissues and high expression on the cell surface of multiple human carcinomas, EpCAM represents an attractive target for immunotherapy ${ }^{10}$.

Solitomab (MT110, AMG 110) is an EpCAM/CD3-bispecific single-chain antibody construct in development for the treatment of patients with multiple solid tumors expressing 
EpCAM $(C D 326)^{11}$. Solitomab mechanism of action is based on the targeting of the EpCAM tumor antigen on tumor cells as well as the recruitment and activation of T-effector cells via CD3 binding. Solitomab has shown remarkable antitumor activity in preclinical ovarian tumor xenograft models due to the ability to engage resting, polyclonal CD8+ and CD4+ T cells for highly potent redirected lysis of target tumor cells.

In this study we report the first evaluation of the in vitro activity of solitomab against multiple primary USC cell lines as well as un-manipulated malignant tumor cells collected from the ascites of patients harboring recurrent-chemotherapy resistant USC. Our results demonstrate impressive solitomab anti-tumor activity against USC cell lines and tumor cells isolated from the ascites of USC patients.

\section{METHODS}

\section{Patients and Sample Processing}

All patients signed an informed consent form according to institutional guidelines and approval for this in vitro study was obtained from the institutional review board. A total of 14 primary USC cell lines were established after sterile processing of surgical biopsy samples as described previously ${ }^{6-8}$. Ascitic fluid samples were collected from two additional patients with cytologically confirmed USC recurrence at the time of a therapeutic paracentesis performed at the time of progression after multiple lines of salvage chemotherapy. Patient characteristics of all USC cell lines and the ascitic fluid effusate are described in Table 1. Primary USC cell lines and freshly collected tumor cell floating in the ascitic fluid were tested for presence of EpCAM-positive uterine cancer cells by flow cytometry as described below.

\section{Ex vivo therapy of malignant ascitic fluid samples}

Malignant ascites from two USC patients were analyzed after ex vivo treatment with solitomab or a control bispecific antibody. The malignant ascites were plated in duplicate in 6-well flat microtiter plate. The ascites was treated with the bispecific antibody construct, solitomab (Amgen Research Munich GmbH, Munich, Germany) at a concentration of $1 \mu \mathrm{g} / \mathrm{ml}$ for 5 days. As a control condition, the ascites were treated with control BiTE® huMEC14 also at a concentration of $1 \mu \mathrm{g} / \mathrm{ml}$. The effect of solitomab on the malignant ascites tumor cells was assessed by observation of induction of morphologic changes and extent of cytotoxicity, as well as, for evidence of $\mathrm{T}$ cell activation and induction of cytokine release as described below.

\section{Flow cytometry}

Characterization of EpCAM expression in malignant ascitic cells before treatment was performed by FACS analysis. The anti-human EpCAM-PE antibody clone 1B7 (eBioscience) was used for flow cytometry studies. The IgG1-PE antibody (BD Biosciences) was used as antibody isotype control for the anti-EpCAM antibody. The detection of the immune cell fractions was determined by using anti-CD8-PE and anti-CD4-PE antibodies. Activation of immune cells was detected by anti-CD25 and anti-HLA-DR antibody. 
Analysis was conducted with FACScalibur flow cytometer with Cell Quest software (Becton Dickinson, Franklin lakes, NJ).

\section{T cell stimulation assay}

Solitomab induced $\mathrm{T}$ cell activation was measured by detecting CD25 protein surface expression and HLA-DR expression on $\mathrm{CD}^{+}$and $\mathrm{CD} 4^{+} \mathrm{T}$ cells by FACS. Solitomab mediated stimulation of $\mathrm{T}$ cells was calculated according to the following formula: Percentage of $\mathrm{CD}^{+} / \mathrm{CD} 25^{+}$expression $=\left[\right.$number of $\mathrm{CD} 8^{+} / \mathrm{CD} 25^{+}$cells $/$total number of

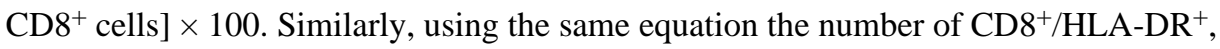
$\mathrm{CD}^{+} / \mathrm{CD} 25^{+}$and $\mathrm{CD} 4+/ \mathrm{HLA}-\mathrm{DR}^{+}$expression was calculated.

\section{Cytokine analysis}

The level of solitomab dependent cytokine induction was compared to the corresponding value of percentage of cytokine release in the control non-specific antibody control wells. This was performed by treating the solitomab and control non-specific antibody wells (at a concentration of $1 \mu \mathrm{g} / \mathrm{ml}$ of solitomab) with phorbol myristate acetate and ionomycin followed by a 3 hour incubation period to allow for lymphocyte stimulation. Brefeldin A was added and a further incubation for 3 hours occurred in order to enhance intracellular cytokine staining signals. Cytokine analysis of the supernatants was performed by FACS analysis after adding anti-CD8-FITC antibody for surface staining followed by fixation, permeabilization and intracellular staining with anti-IL-4-PE antibody and anti-IFN gammaPE antibody. Solitomab mediated release of each of these cytokines was calculated according to the following exemplary formula: Percentage of $\mathrm{CD}^{+} / \mathrm{IFN}$ gamma containing cells $=\left[\right.$ number of $\mathrm{CD}^{+} /$IFN gamma cells/ total number of $\mathrm{CD}^{+}$cells $] \times 100$. Similar calculations were performed for $\mathrm{CD} 4{ }^{+} \mathrm{T}$ cells (i.e., gated $\mathrm{CD} 3+/ \mathrm{CD} 8-\mathrm{T}$ cells).

\section{Proliferation assay of tumor associated T-lymphocytes (TAL) after the addition of EpCAM BiTE $®$ by CFSE Cell proliferation}

Briefly, cells were harvested from ascites samples and washed twice with PBS and immediately stained with carboxyfluorescein succinimidyl ester (CFSE) (CellTrace CFSE Cell Proliferation Kit, Invitrogen, Carlsbad, CA) at a working concentration of 10 micromolar. The CFSE labeled cells were plated and cultured in the presence of Control BiTE® huMEC14 or solitomab for 5 days. Cells were collected and labeled with CD8 and CD4 (Becton Dickinson) and analyzed on a flow cytometer (FacsCalibur, Becton Dikinson) using Cell Quest software. The percentage and numbers of CD8+ and CD4+ T cells in the control BiTE® wells vs solitomab treated wells were calculated after analysis by flow cytometry to determine TAL proliferation induced in this co-culture system.

\section{Tests for T cell mediated cytotoxicity}

The standard 4-hour chromium $\left({ }^{51} \mathrm{Cr}\right)$ release assay was used to measure the cytotoxic reactivity of Ficoll-Hypaque-separated peripheral blood lymphocytes (PBLs) from several healthy donors against five representative USC cell lines at effector to target ratios (E:T) of 10:1 and 20:1. The release of ${ }^{51} \mathrm{Cr}$ from target cells was measured as evidence of tumor cell lysis after exposure of the tumor cells to a concentration of $1 \mu \mathrm{g} / \mathrm{ml}$ of solitomab. The 
negative control conditions were the incubation of target cells alone or with PBL without BiTE® antibody construct. As a positive control condition, $1 \%$ sodium dodecyl sulfate (SDS) was used to achieve complete lysis of target cells. The control BiTE® huMEC14 at $1 \mu \mathrm{g} / \mathrm{ml}$ was used as the negative control for solitomab in this bioassay. The control BiTE® antibody construct shared the CD3 binding arm with solitomab, but otherwise recognizes an herbicide as an irrelevant antigen instead of recognizing EpCAM. The percentage cytotoxicity of solitomab was calculated by the following formula: $\%$ cytotoxicity $=100 \times$ $(E-S) / T-S)$, where $E$ is the experimental release, $S$ is the spontaneous release by effector cells, $T$ is the maximum release by target cells lysed with $1 \%$ SDS.

\section{STATISTICS}

Differences in EpCAM expression by flow cytometry were analyzed by the unpaired $t$ test. The unpaired $t$ test was used to evaluate the differences in ADCC levels in primary tumor cell lines treated with solitomab versus control BiTE® huMEC14. T cell activation marker increase as well as cytokine release was analyzed by the paired $t$ test. Statistical analysis was performed using SPSS version 18. A P-value of $<0.05$ was considered as the level of statistical significance.

\section{RESULTS}

\section{Epithelial cell adhesion molecule levels in primary USC cell lines and ascitic samples}

We used flow cytometry to obtain highly sensitive measurements of EpCAM surface expression in 14 primary USC lines ( 2 established from patients harboring chemotherapy resistant disease) ${ }^{12,13}$ and in 2 ascitic fluid samples collected through paracentesis performed at the time of tumor progression after multiple regimens of chemotherapy. Table 1 depicts the characteristics of the patients from which the samples were collected and shows the percentage of EpCAM positive cells and corresponding mean fluorescence intensity (MFI) in primary USC cell lines and ascitic fluid samples by flow cytometry. Amongst the USC primary cell lines, 12 out of 14 (85.7\%) were found to express EpCAM in $100 \%$ of the tumor cells (Table 1 and Figure 1). EpCAM expression levels were highest in USPC-ARK-22 specimens (both in the primary cell line tested after multiple passages in vitro as well as in the freshly collected tumor cells floating in patient's ascites collected at the time of tumor progression, Table 1). In contrast, lower EpCAM expression was found in USPC-ARK-4, USPC-ARK-7, USPC-ARK-11 and USPC-ARK-24.

\section{USC primary cell lines are resistant to natural killer (NK) cell activity but sensitive to solitomab-mediated $\mathrm{T}$ cell cytotoxicity}

Standard 4-hour chromium $\left({ }^{51} \mathrm{Cr}\right)$ release assay cytotoxicity titration was performed using solitomab (ЕрCAM BiTE®) from doses of 0 to $2.5 \mu \mathrm{g} / \mathrm{ml}$ in several experiments aimed at determining the optimum dose of solitomab against a representative USC. From these experiments the $1 \mu \mathrm{g} / \mathrm{ml}$ concentration emerged as the optimum concentration for tumor killing (Figure 2, upper panel). Next, five representative primary uterine cancer cell lines were evaluated for their sensitivity to NK and T-cells. These USC cell lines were exposed to peripheral blood lymphocytes collected from multiple healthy donors in several cytotoxicity assays. These peripheral blood lymphocytes typically contain between $20-25 \% \mathrm{CD} 8^{+} \mathrm{T}$ 
cells and 10-20\% NK cells (CD56 $\left.{ }^{+} / \mathrm{CD} 16^{+}\right)$. USC cell lines were resistant to both NK and T-cell-mediated killing with exposure to PBL at effector: target ratios of 1:10 and 1:20 (mean killing \pm STDEV, $2.7 \pm 3.1 \%$ with a range of $0-8.4 \%$ ) after incubation of EpCAMpositive cell lines with control BiTE® at a $1: 20$ ratio) (Figure 2, lower panel). In contrast, significant tumor kill was seen when EpCAM positive cell lines were incubated with solitomab. Addition of solitomab modulated T cell mediated cytotoxicity with a range of killing of 20.3-34.4\%; mean killing \pm STDEV, $25.7 \pm 4.5 \%$; P $<0.0001$. As expected, the low EpCAM expressing cell line (USPC-ARK-4) demonstrated negligible levels of cytotoxicity with the addition of solitomab compared with other USC cell lines and control BiTE® (Figure 2, lower panel).

\section{Co-culture of solitomab (EpCAM BiTE®) and TAL in ascitic fluid triggers T-lymphocyte proliferation and autologous tumor-cell cytotoxicity}

T-lymphocyte proliferation and cytotoxicity was evaluated after co-culturing EpCAMpositive tumor cells with solitomab and autologous TAL in two freshly collected ascitic fluid samples (i.e., USPC-ARK19 and USPC-ARK-22) for 5 days. CFSE as well as antiCD4 and anti-CD8 antibodies were used to label lymphocytes and anti-EpCAM antibodies to label tumor cells to be counted by flow cytometry. Incubation with solitomab, compared to control BiTE®, significantly increased lymphocyte proliferation in both CD8 ${ }^{+} \mathrm{T}$ cells and $\mathrm{CD} 4^{+} \mathrm{T}$ numbers in the ascitic fluid culture. These results were validated through multiple experiments (upper panel, Figure 3, P $<0.005$ ). The activated T-lymphocytes primed for tumor cell destruction by solitomab also had a change in their morphologic appearance, as T-lymphocytes in the solitomab co-culture appeared larger than in the control BiTE® (data not shown). Next, the ability of solitomab to stimulate lymphocyte cytotoxic activity against minimally manipulated USC cells floating as free tumor cells or spherocytes in ascitic fluid was evaluated. Incubation for 5 days with solitomab significantly decreased the number of EpCAM-positive tumor cells in the freshly collected USPC-ARK-19 and USPC-ARK-22 ascitic fluid samples (both derived from two patients harboring recurrent chemotherapyresistant disease). The lower panel of Figure 3 depicts the decrease of EpCAM-positive tumor cells vs control BiTE® after 5 day incubation (mean + STDEV $=29.1 \%+5.1$ for USPC-ARK-19 and $2.55+0.5 \%$ for USPC-ARK-22 p<0.0001).

\section{Activation of T- lymphocytes by solitomab (EpCAM BiTE®) in co-cultures with USC cells}

Next, we explored whether solitomab is capable of inducing $\mathrm{T}$ cell activation in the presence of uterine serous tumor cells in ascitic fluid samples. $\mathrm{CD} 8^{+}$and $\mathrm{CD} 4^{+} \mathrm{T}$ cells at baseline and after 5-day co-cultures with ascitic fluid and solitomab were analyzed by flow cytometry for the expression of the T-cell activation markers CD25 and HLA-DR. As demonstrated in the upper panel of Figure 4, we found a significant increase in both CD25 and HLA-DR protein surface expression on $\mathrm{CD}^{+}$and $\mathrm{CD} 4^{+} \mathrm{T}$ cells in the solitomab treated wells when compared to the wells treated with the control BiTE® $(\mathrm{p}<0.001)$.

\section{Cytokine release accompanying $\mathrm{T}$ lymphocyte activation}

The level of the solitomab dependent cytokine induction was compared to the cytokine release in the control non-specific BiTE® control wells. The flow analysis was performed after 5 days of co-incubation of solitomab with tumor cells and TAL before lymphocyte 
stimulation in the presence of brefeldin A. Analysis by FACS revealed a significant increase in IFN $-\gamma$ production, by both CD4+ T cells and CD8+ T cells, in EpCAM + ascites exposed to solitomab compared to control BiTE®, $\mathrm{P}<0.005$ (Figure 4, lower panel). In contrast, no significant increase in IL-4 release was noted in solitomab exposed ascitic T-cells when compared to wells stimulated with control BiTE® ( $\mathrm{p}=0.1$, Figure 4, lower panel).

\section{DISCUSSION}

Our research group has recently reported on the expression profile and genetic landscape of uterine serous carcinoma ${ }^{6-8}$. The gene encoding EpCAM/TROP-1 was consistently identified as one of the most up-regulated genes in $\mathrm{USC}^{6-8}$. EpCAM expression is highest during embryogenesis and elevated expression has been reported in a number of different carcinomas ${ }^{1415}$. Normal mature tissues generally show very low expression of EpCAM on the basolateral surface of epithelia ${ }^{14,15}$. Due to its restricted accessibility in highly structured epithelium, normal physiologic EpCAM is considered minimally accessible, at best, to intravenously administered anti-EpCAM mAb. This has been demonstrated by comparing antibody accessibility to human EpCAM-expressing syngeneic tumors and normal epithelial tissues in transgenic mice ${ }^{16}$. In contrast, the high density expression and accessibility of EpCAM on metastatic/chemotherapy-resistant USC make EpCAM a promising target for BiTE®-based therapy in patients with USC refractory to standard treatment modalities.

BiTE® molecules are bispecific antibody constructs able to form a cytolytic synapse between tumor cells and $\mathrm{T}$ cells bringing them into close proximity. By bridging cancer cells to the CD3 T cell-receptor directly, these responses are not restricted by $\mathrm{T}$ cell receptor specificity, presence of MHC class I, generation and presentation of peptide antigen, or the need for T cell co14 stimulation. In this study we explored the potential of solitomab, an EpCAM/CD3 bispecific antibody construct (BiTE®), as a novel therapeutic strategy against chemotherapy-resistant USC. First, we evaluated EpCAM expression in multiple primary USC cell lines obtained from patients with advanced and chemotherapy resistant disease as well as in free tumor cells and spheroids formed in vivo collected from USC patients. Consistent with our previous results in ovarian cancer $^{12,13}$, we found that EpCAM was overexpressed in the majority of the primary cell lines. Importantly, we also found primary USC cell lines overexpressing EpCAM to be highly susceptible to ADCC mediated by solitomab. The activity of solitomab against multiple human tumors including colon cancer as well as ovarian cancer has previously been demonstrated by our group as well as others ${ }^{17,18}$. In this study, we extend these results by evaluating the cytotoxic potential of solitomab against multiple biologically aggressive and highly chemotherapy-resistant uterine serous cancer cell lines. All primary USC tested with significant EpCAM surface expression were found highly susceptible to solitomab-mediated $\mathrm{T}$ cell killing in the presence of effector cells while negligible cytotoxicity was detected in cell lines with low level of EpCAM expression.

Natural killer cells play an important role in surveillance and elimination of cancer. In our experiments, EpCAM-positive cell lines were found to be highly resistant to natural killer cytotoxic activity. In contrast, solitomab-mediated T cell cytoxicity resulted in killing of up to $34.4 \%$ of cells in 4 -hour ${ }^{51} \mathrm{Cr}$ release assays. Importantly, uterine serous tumor cells 
present as single cell suspensions or as tumor spheroids isolated from the ascites of USC patients harboring chemotherapy resistant disease were also highly susceptible to solitomabmediated cytotoxicity. Because our results were obtained by adding solitomab to the freshly collected tumor cells and TAL present in the ascitic fluid in the absence of any immunestimulatory cytokines we believe these data may serve as a preview of the effects that solitomab may have on recurrent disease in vivo, particularly when administered via an intra-peritoneal route.

Our study also shows that solitomab is highly active at low effector to target ratios and that in addition to triggering cytotoxicity, solitomab is also capable of inducing significant CD8+ and CD4+ T lymphocyte proliferation, expression of activation markers and Type I cytokine production in both autologous CD8+ and CD4+ TAL present in the ascitic fluid of a heavily pretreated USC patients. Data from ex-vivo experiments with minimally manipulated EpCAM positive tumor cells demonstrate that autologous TAL are able to significantly reduce the size and integrity of highly chemotherapy-resistant uterine tumor spheroids floating in the ascitic fluid when exposed to solitomab. These results are highly encouraging considering that a large number of studies have revealed that TIL and TAL in the tumor tissues or ascitic fluid are frequently anergic and/or tolerogenic against autologous tumor cells in a variety of human tumors ${ }^{19-22}$. Taken together, these data suggest that even partially tumor- anergic $\mathrm{T}$ cells in ascitic fluid may become activated by solitomab and mount a cytotoxic T-cell response able to kill chemotherapy resistant USC cells.

The widespread expression and membranous localization of EpCAM in USC combined with its negative expression in mesothelial type cells in the abdominal cavity, suggests that this protein could represent an accessible tumor target antigen for both intravenous and intraperitoneal antibody/BiTE®-based therapies. Consistent with this view, a phase 1 study of EpCAM/CD3-bispecific antibody (MT110) in patients harboring advanced tumors is currently ongoing ${ }^{23}$. The first clinical intraperitoneal application of a trifunctional antiEpCAM antibody (catumaxomab/Removab®) has recently shown effective tumor cell destruction, substantially decreased ascites accumulation, and reduced necessity of paracentesis in advanced ovarian carcinoma patients harboring malignant tumors refractory to salvage chemotherapy ${ }^{24}$. As a result, catumaxomab has recently received market approval by the European Medicines Agency (EMA) for this indication. Importantly, Blinatumomab, a bispecific CD19-directed CD3 T-cell engager (BiTE®) has recently obtained FDA's breakthrough therapy designation and accelerated approval for the treatment of patients with Philadelphia chromosome-negative (Ph-) relapsed or refractory B-cell precursor acute lymphoblastic leukemia (ALL) 252627.

In conclusion, this is the first report of solitomab therapeutic activity in USC, the most aggressive and chemotherapy resistant variant of endometrial cancer. Our findings demonstrate that: a) twelve out of fourteen primary tumor cell lines derived from patients harboring advanced stages USC expressed EpCAM on their cell surfaces as measured by flow cytometry, b) primary USC cell lines overexpressing EpCAM are highly susceptible to ADCC mediated by solitomab, an EpCAM/CD3 bispecific antibody construct (BiTE®) and c) incubation with solitomab when compared to control BiTE® may significantly increase $\mathrm{CD}^{+}$and $\mathrm{CD} 8^{+} \mathrm{T}$ cell numbers as well as the activation, Type I cytokine secretion and 
cytotoxic activity of TAL in the ascitic fluid of USC patients. The future design and implementation of clinical trials in this regard will ultimately determine the validity of this novel therapeutic approach in patients harboring USC.

\title{
Acknowledgments
}

\author{
Financial Support:
}

This work was supported in part by grants from NIH R01 CA154460-01A1 and U01 CA176067-01A1, the Honorable Tina Brozman Foundation, the Deborah Bunn Alley Ovarian Cancer Research Foundation, the Guido Berlucchi Research Foundation and the Discovery to Cure Foundation to ADS. This investigation was also supported by NIH Research Grant CA-16359 from the NCI.

\section{REFERENCES}

1. Siegel RL, Miller KD, Jemal A. Cancer statistics, 2015. CA: a cancer journal for clinicians. 2015; 65:5-29. [PubMed: 25559415]

2. Hendrickson M, Ross J, Eifel P, Martinez A, Kempson R. Uterine papillary serous carcinoma: a highly malignant form of endometrial adenocarcinoma. Am J Surg Pathol. 1982; 6:93-108. [PubMed: 7102898]

3. Slomovitz BM, Burke TW, Eifel PJ, et al. Uterine papillary serous carcinoma (UPSC): a single institution review of 129 cases. Gynecologic oncology. 2003; 91:463-469. [PubMed: 14675663]

4. Goff BA, Kato D, Schmidt RA, et al. Uterine papillary serous carcinoma: patterns of metastatic spread. Gynecol Oncol. 1994; 54:264-268. [PubMed: 8088602]

5. Nicklin JL, Copeland LJ. Endometrial papillary serous carcinoma: patterns of spread and treatment. Clin Obstet Gynecol. 1996; 39:686-695. [PubMed: 8862892]

6. Zhao S, Choi M, Overton JD, et al. Landscape of somatic single-nucleotide and copynumber mutations in uterine serous carcinoma. Proc Natl Acad Sci U S A. 2013; 110:2916-2921. [PubMed: 23359684]

7. Santin AD, Zhan F, Bellone S, et al. Discrimination between uterine serous papillary carcinomas and ovarian serous papillary tumours by gene expression profiling. British journal of cancer. 2004; 90:1814-1824. [PubMed: 15208622]

8. Santin AD, Zhan F, Cane S, et al. Gene expression fingerprint of uterine serous papillary carcinoma: identification of novel molecular markers for uterine serous cancer diagnosis and therapy. British journal of cancer. 2005; 92:1561-1573. [PubMed: 15785748]

9. Munz M, Baeuerle PA, Gires O. The emerging role of EpCAM in cancer and stem cell signaling. Cancer research. 2009; 69:5627-5629. [PubMed: 19584271]

10. Armstrong A, Eck SL. EpCAM: A new therapeutic target for an old cancer antigen. Cancer biology \& therapy. 2003; 2:320-326. [PubMed: 14508099]

11. Brischwein K, Schlereth B, Guller B, et al. MT110: a novel bispecific single-chain antibody construct with high efficacy in eradicating established tumors. Molecular immunology. 2006; 43:1129-1143. [PubMed: 16139892]

12. Bellone S, Siegel ER, Cocco E, et al. Overexpression of epithelial cell adhesion molecule in primary, metastatic, and recurrent/chemotherapy-resistant epithelial ovarian cancer: implications for epithelial cell adhesion molecule-specific immunotherapy. International journal of gynecological cancer : official journal of the International Gynecological Cancer Society. 2009; 19:860-866. [PubMed: 19574774]

13. Richter CE, Cocco E, Bellone S, et al. High-grade, chemotherapy-resistant ovarian carcinomas overexpress epithelial cell adhesion molecule (EpCAM) and are highly sensitive to immunotherapy with MT201, a fully human monoclonal anti-EpCAM antibody. American journal of obstetrics and gynecology. 2010; 203:582 e1-582 e7. [PubMed: 20870202]

14. Balzar M, Winter MJ, de Boer CJ, Litvinov SV. The biology of the 17-1A antigen (Ep-CAM). Journal of molecular medicine. 1999; 77:699-712. [PubMed: 10606205] 
15. Spurr NK, Durbin H, Sheer D, Parkar M, Bobrow L, Bodmer WF. Characterization and chromosomal assignment of a human cell surface antigen defined by the monoclonal antibody AUAI. International journal of cancer Journal international du cancer. 1986; 38:631-636. [PubMed: 3770992]

16. McLaughlin PM, Harmsen MC, Dokter WH, et al. The epithelial glycoprotein 2 (EGP-2) promoter-driven epithelial-specific expression of EGP-2 in transgenic mice: a new model to study carcinoma-directed immunotherapy. Cancer research. 2001; 61:4105-4111. [PubMed: 11358833]

17. Schlereth B, Fichtner I, Lorenczewski G, et al. Eradication of tumors from a human colon cancer cell line and from ovarian cancer metastases in immunodeficient mice by a single-chain EpCAM-/CD3-bispecific antibody construct. Cancer research. 2005; 65:2882-2889. [PubMed: 15805290]

18. English DP, Bellone S, Schwab CL, et al. Solitomab, an epithelial cell adhesion molecule/CD3 bispecific antibody (BiTE), is highly active against primary chemotherapy-resistant ovarian cancer cell lines in vitro and fresh tumor cells ex vivo. Cancer. 2015; 121:403-412. [PubMed: 25251053]

19. Radoja S, Saio M, Schaer D, Koneru M, Vukmanovic S, Frey AB. CD8(+) tumor-infiltrating T cells are deficient in perforin-mediated cytolytic activity due to defective microtubule-organizing center mobilization and lytic granule exocytosis. Journal of immunology (Baltimore, Md: 1950). 2001; 167:5042-5051.

20. Bocchia M, Bronte V, Colombo MP, et al. Antitumor vaccination: where we stand. Haematologica. 2000; 85:1172-1206. [PubMed: 11074658]

21. Costello RT, Gastaut JA, Olive D. Tumor escape from immune surveillance. Archivum immunologiae et therapiae experimentalis. 1999; 47:83-88. [PubMed: 10202560]

22. Sinkovics JG, Horvath JC. Vaccination against human cancers (review). International journal of oncology. 2000; 16:81-96. [PubMed: 10601552]

23. Walter, M.; Fiedler, MW.; Maxim, Kebenko; Marie-Elisabeth, Goebeler; Barbara, Ritter; Alexander, Quaas, et al. Oral Abstract Session, Developmental Therapeutics - Clinical Pharmacology and Immunotherapy. ASCO Annual Meeting; 2012. A phase I study of EpCAM/ CD3-bispecific antibody (MT110) in patients with advanced solid tumors. Oral Abstract Session

24. Burges A, Wimberger P, Kumper C, et al. Effective relief of malignant ascites in patients with advanced ovarian cancer by a trifunctional anti-EpCAM $\mathrm{x}$ anti-CD3 antibody: a phase I/II study. Clinical cancer research : an official journal of the American Association for Cancer Research. 2007; 13:3899-3905. [PubMed: 17606723]

25. Topp MS, Gokbuget N, Zugmaier G, et al. Phase II trial of the anti-CD19 bispecific T cell-engager blinatumomab shows hematologic and molecular remissions in patients with relapsed or refractory B-precursor acute lymphoblastic leukemia. Journal of clinical oncology : official journal of the American Society of Clinical Oncology. 2014; 32:4134-4140. [PubMed: 25385737]

26. Sanford M. Blinatumomab: first global approval. Drugs. 2015; 75:321-327. [PubMed: 25637301]

27. Oak E, Bartlett NL. Blinatumomab for the treatment of B-cell lymphoma. Expert opinion on investigational drugs. 2015:1-10. 


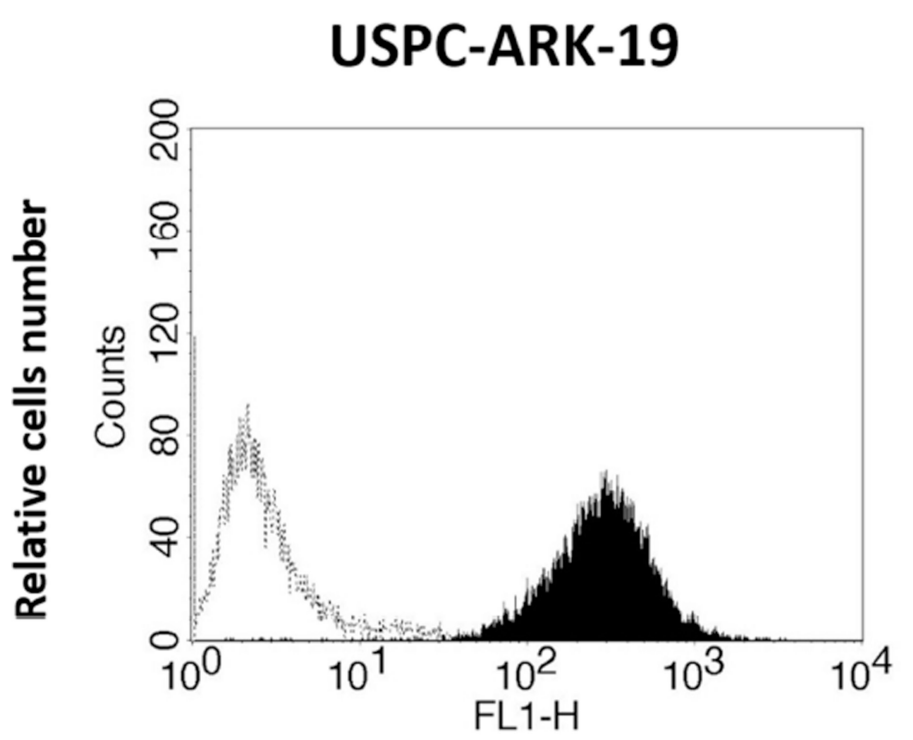

EpCAM/TROP-1

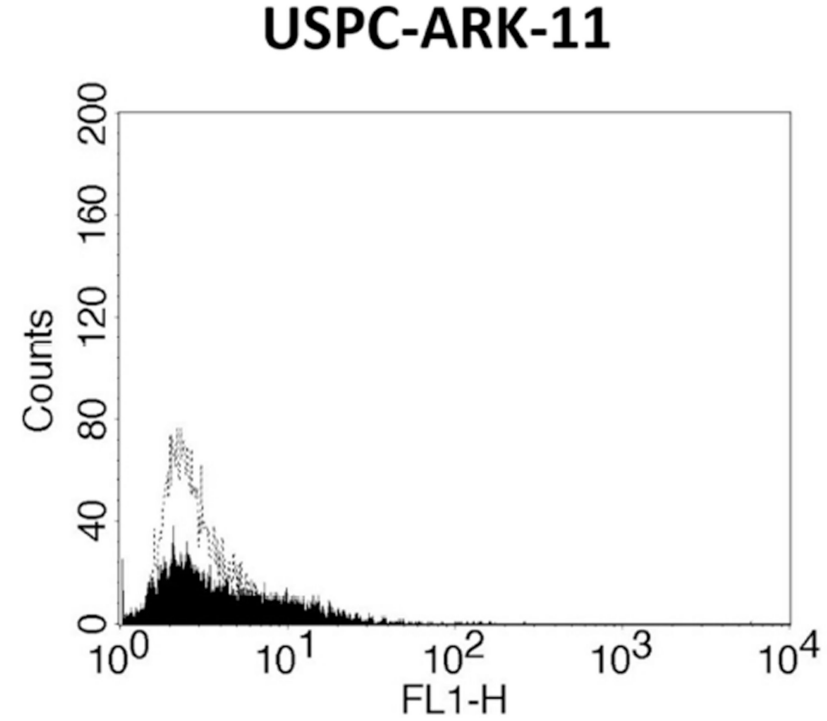

EpCAM/TROP-1

Figure 1.

Flow cytometry histograms of two representative primary USC cell lines showing high (i.e., USPC-ARK-19) vs low/negligible (USPC-ARK-11) expression of EpCAM. Dashed lines indicate isotypes. 
CYTOTOXCITY TITRATION ARK-2

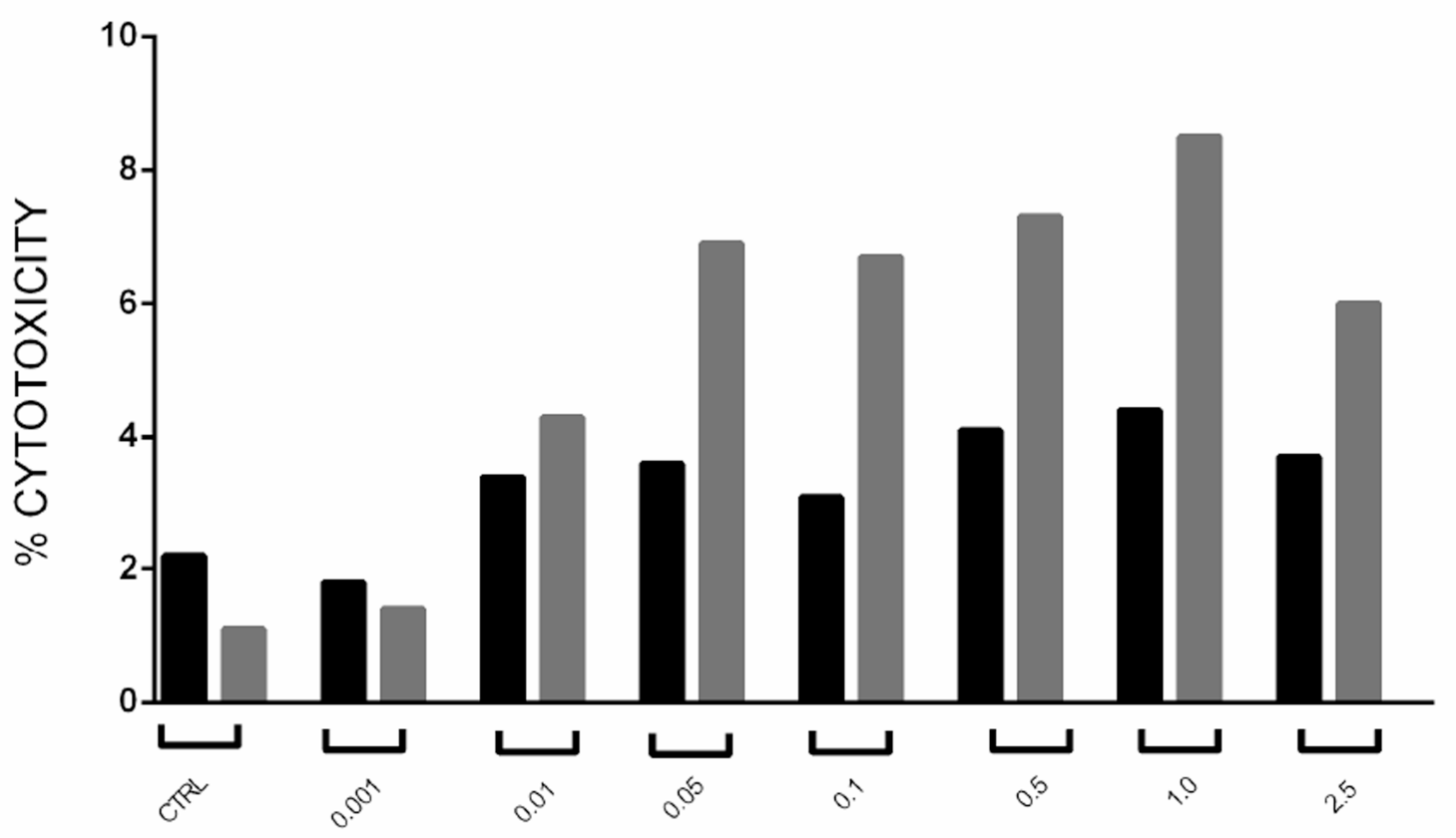

CYTOTOXCITY IN USPC CELL LINES

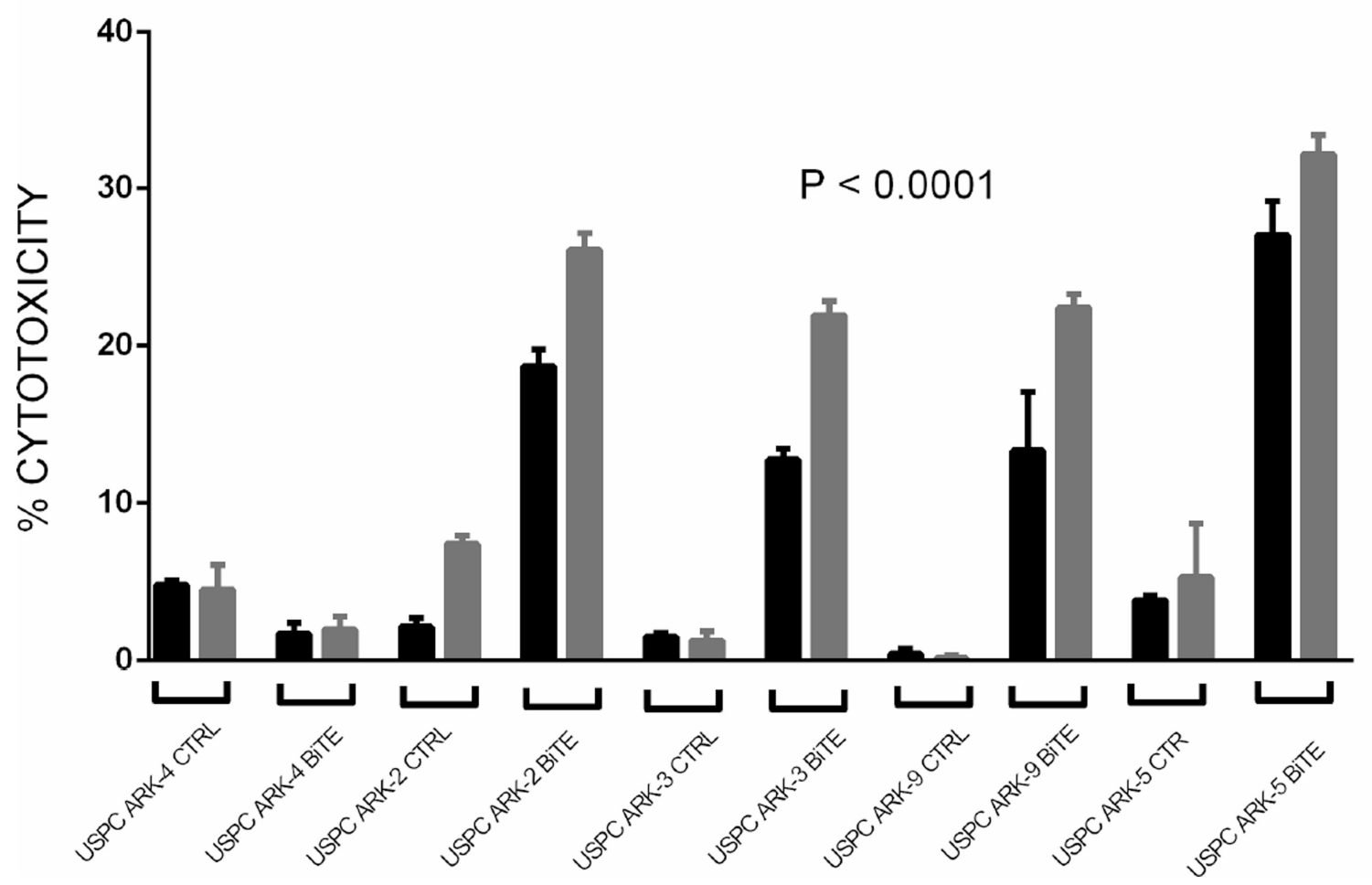

Figure 2.

Upper Panel. Solitomab (EpCAM BiTE®) cytotoxicity titration graph for USPC-ARK-2. Doses from 0 to $2.5 \mu \mathrm{g} / \mathrm{ml}$ were used in several cytotoxicity experiments aimed at 
determining the optimum dose of solitomab. As a results of these experiments the $1 \mu \mathrm{g} / \mathrm{ml}$ concentration was selected as optimal for the additional cytotoxicity assays. Lower Panel. Graph showing antibody-dependent cell-mediated cytotoxicity for five representative USC cell lines in control conditions and with solitomab at two effector: target ratios. As expected, the higher effector: target ratio of 1:20 (i.e., the ratio of T lymphocyte effector cells vs number of tumor target cell used in the assay) demonstrated higher cytotoxicity results. 
Proliferation assay

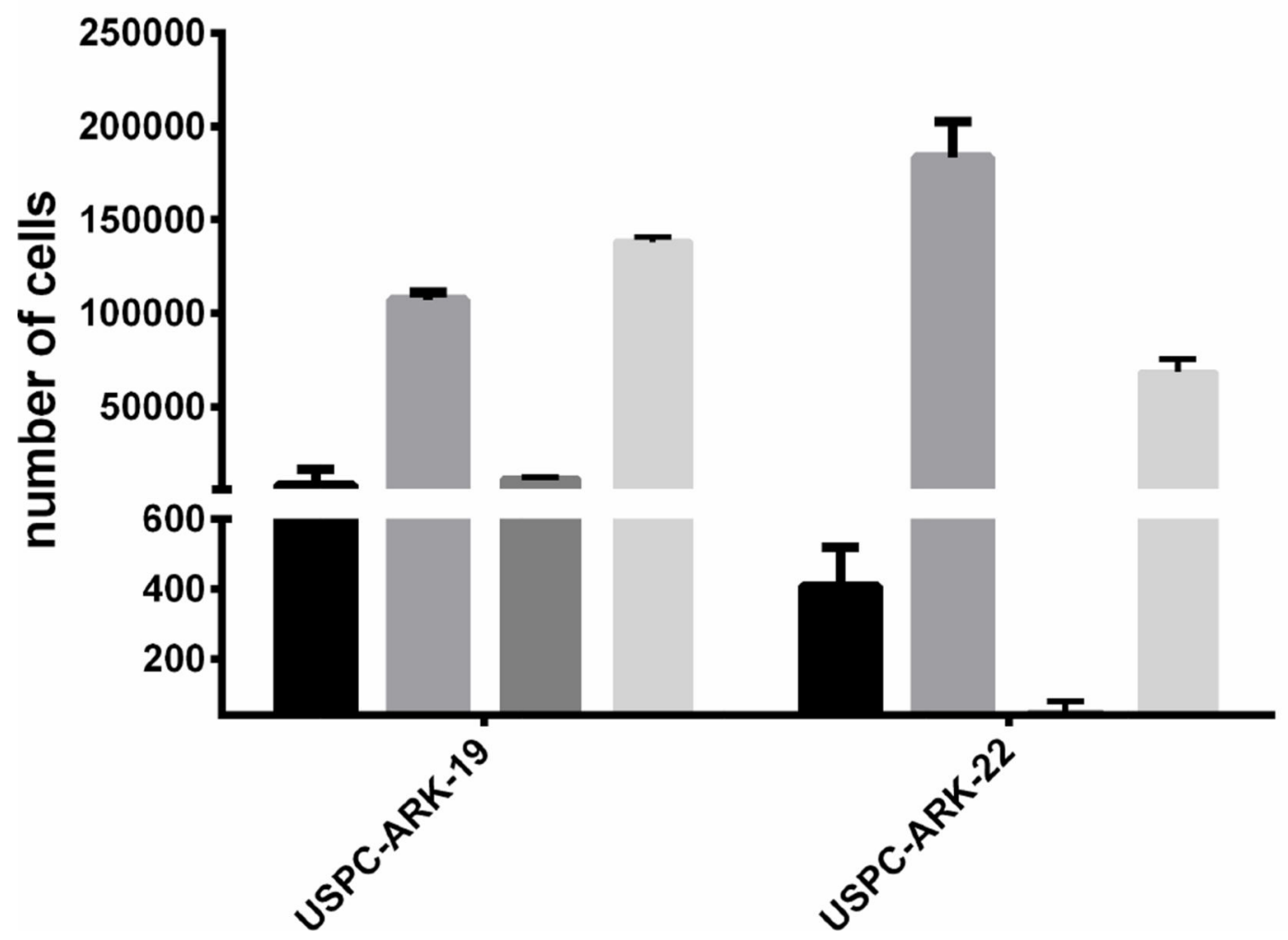




\section{Viability assay}

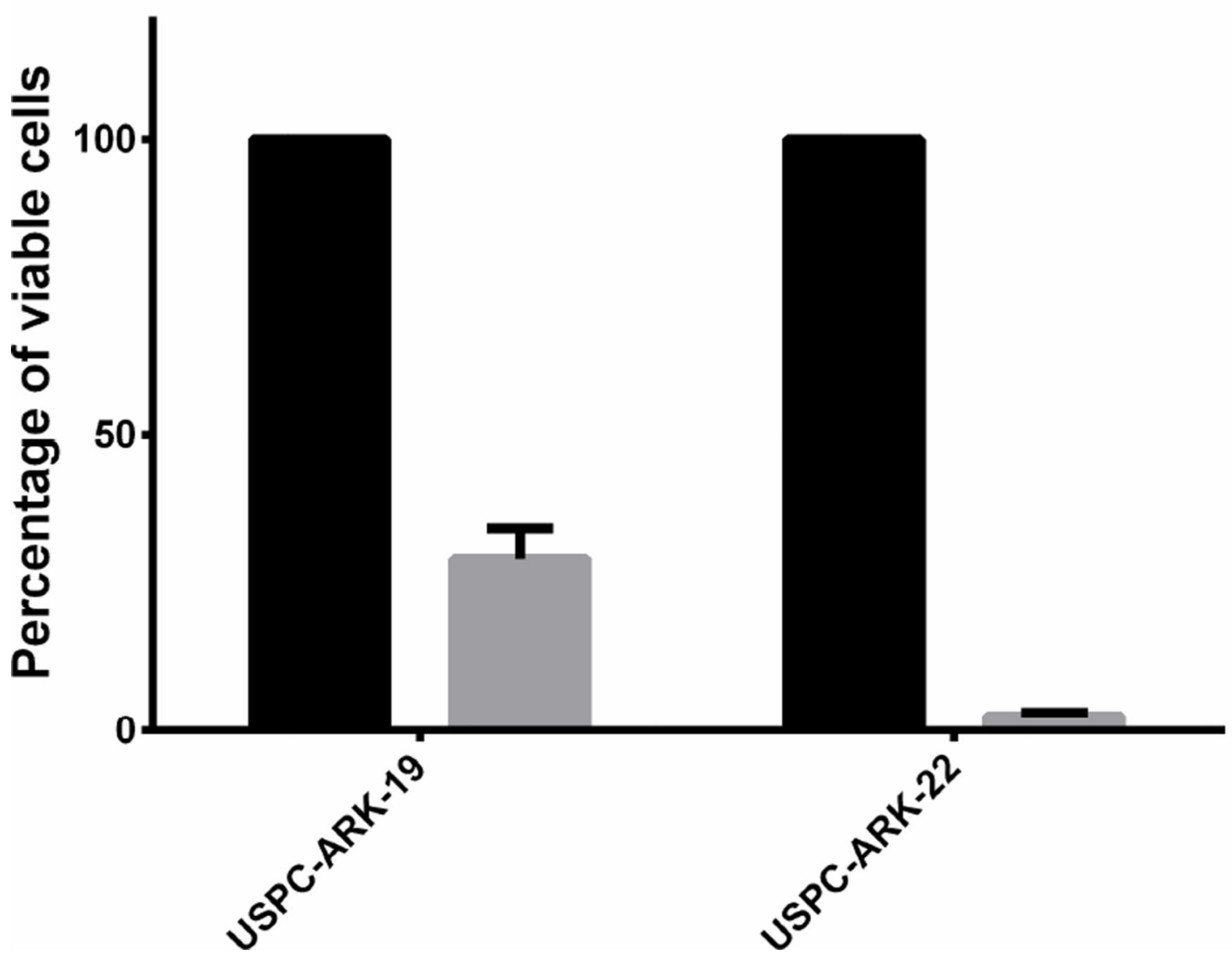

Figure 3.

Upper Panel. $\mathrm{CD} 8^{+}$and $\mathrm{CD} 4^{+} \mathrm{T}$ cell proliferation in the ascitic fluid of EpCAM + USC patients after incubation with control BiTE® vs solitomab. Lower panel. Graph showing the percentage of EpCAM positive viable cell in patients' ascites after 5 days incubation with control BiTE® vs solitomab. 
Activation markers USPC-ARK-19

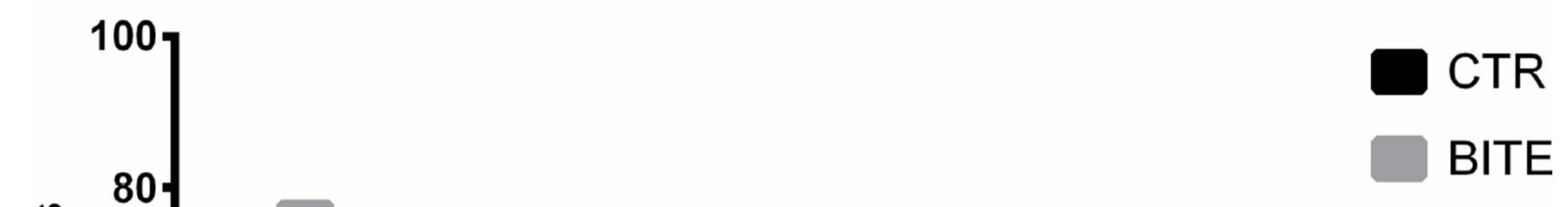

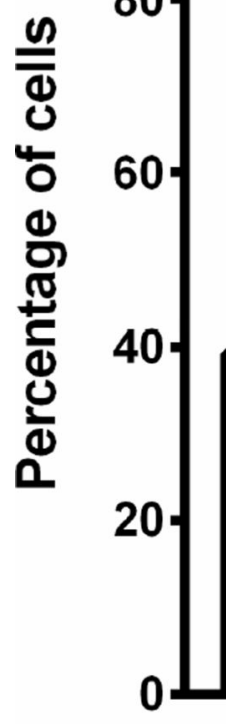

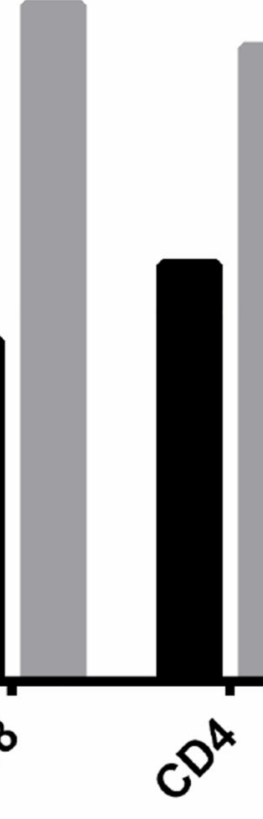<smiles>C1CCCCCCOCCCCCC1</smiles><smiles>[O+][O-]</smiles><smiles>Oc1ccoc1</smiles>

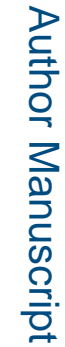

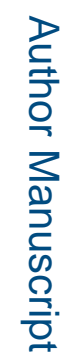


Activation markers USPC-ARK-22

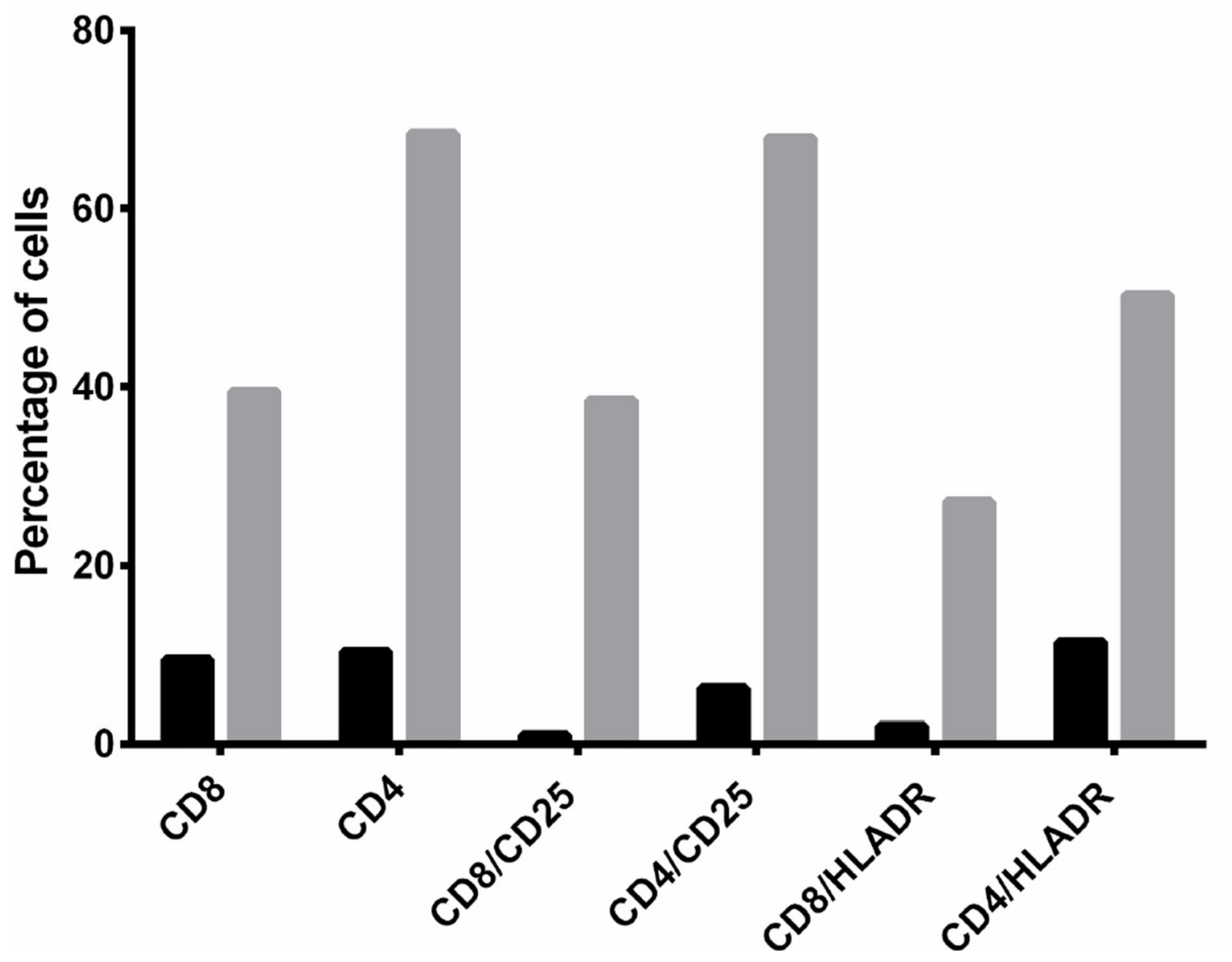

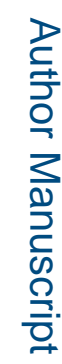


Cytokines USPC-ARK-19

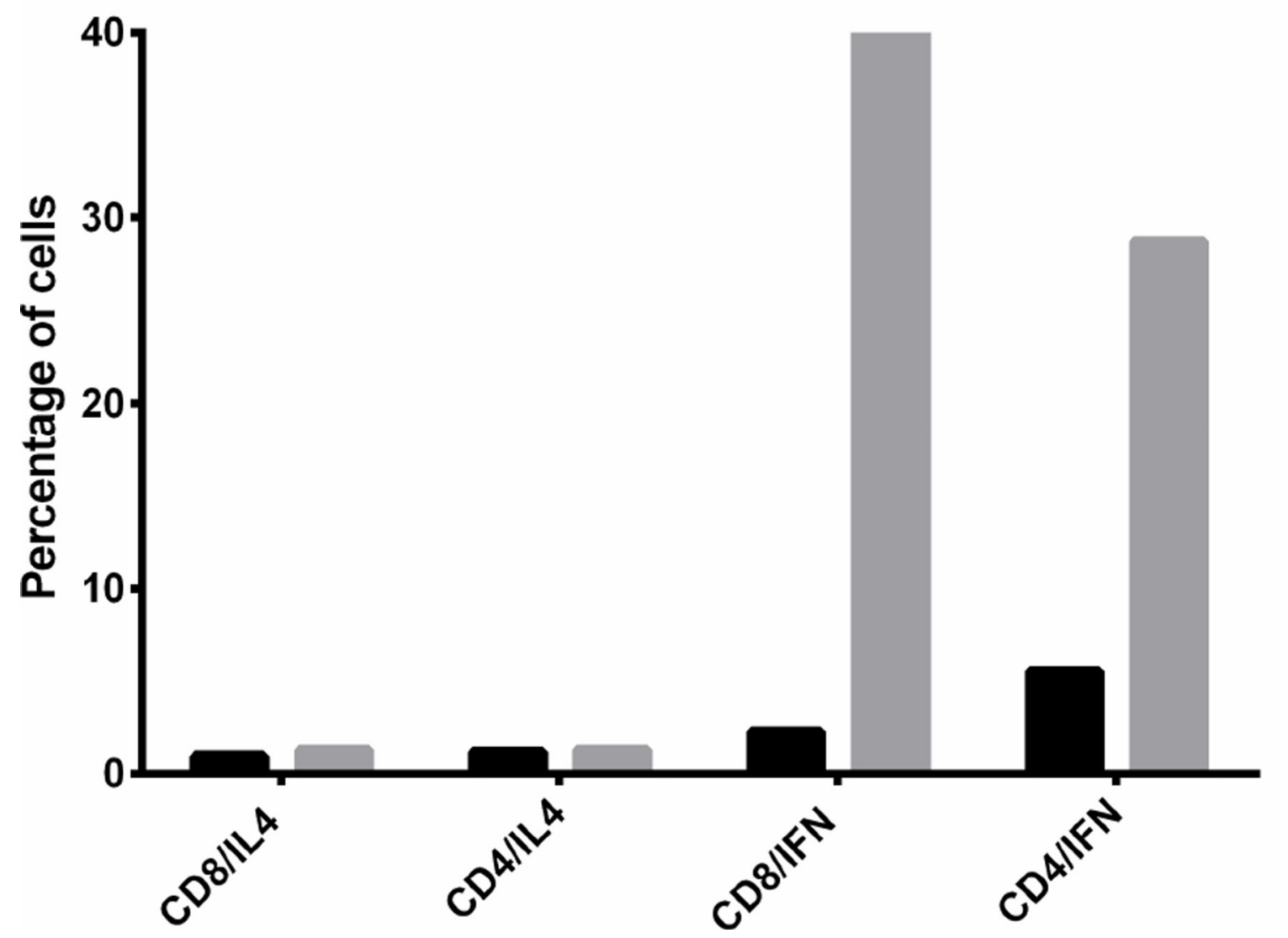




\section{Cytokines USPC-ARK-22}

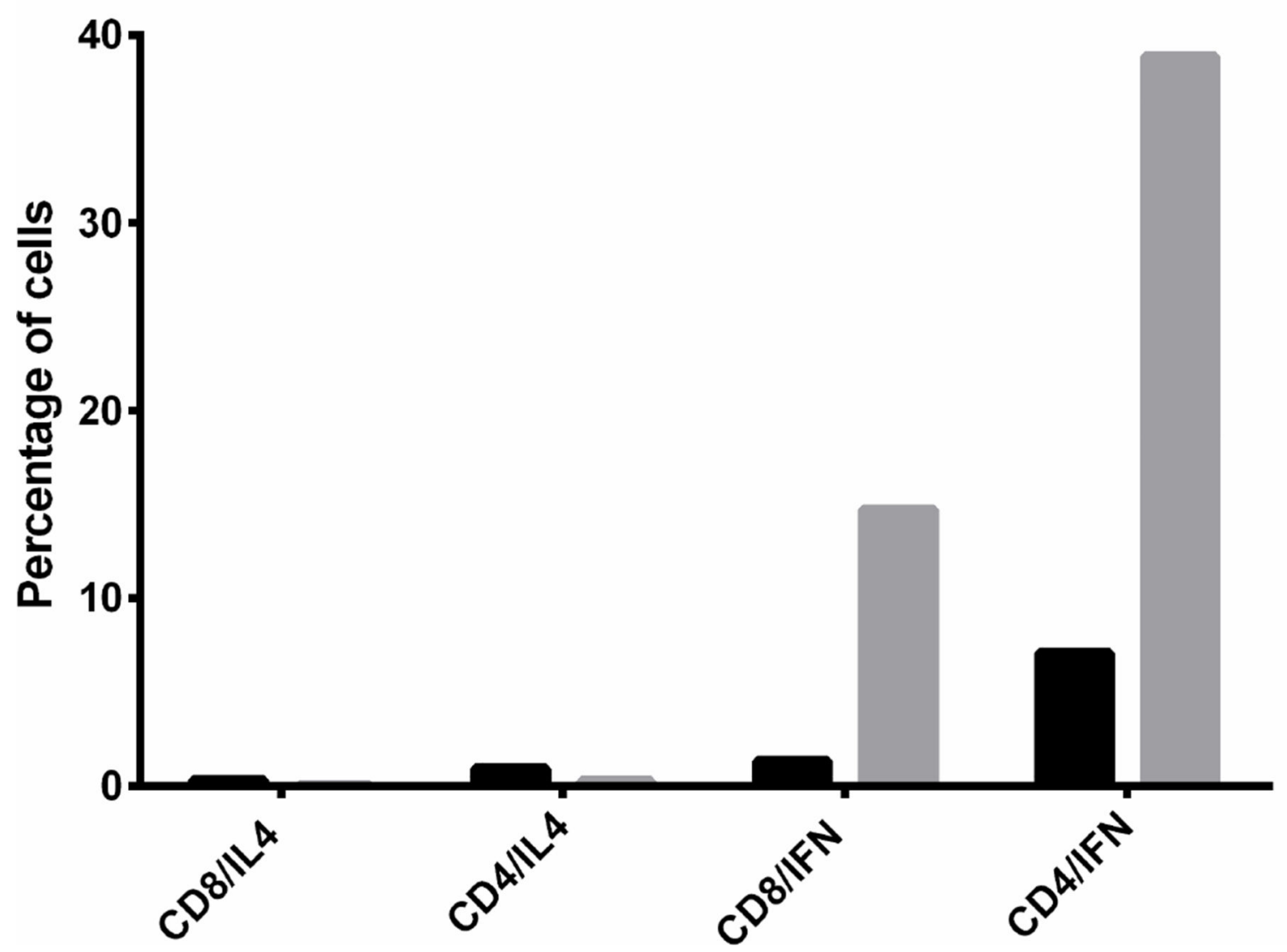

Figure 4.

Upper panels. CD25 and HLA-DR activation marker expression in $\mathrm{CD}^{+}$and $\mathrm{CD}^{+}{ }^{+} \mathrm{T}$ cells after stimulation of T-lymphocytes exposed to solitomab vs control BiTE®. A significant increase in CD25 and HLA-DR activation marker expression was consistently detected in both $\mathrm{CD}^{+}$or $\mathrm{CD}^{+} \mathrm{T}$ cells. Lower panels. Representative IFN-gamma cytokine release after stimulation of T-lymphocytes exposed to solitomab vs control BiTEß. A significant increase in IFN-gamma stained cells was consistently detected in both $\mathrm{CD}^{+}$or $\mathrm{CD}^{+} \mathrm{T}$ cells. 


\begin{tabular}{cccc}
\hline $\begin{array}{c}\text { Tumor cell line } \\
\text { designation }\end{array}$ & Histology & \multicolumn{2}{c}{$\begin{array}{c}\text { Binding of } \\
\text { Ep-CAM Mab }\end{array}$} \\
\cline { 3 - 4 } & & \% cells & MFI \\
\hline USPC-ARK-1 & Pure & 100 & 89 \\
USPC-ARK-2 & Pure & 100 & 124 \\
USPC-ARK-3 & Pure & 100 & 171 \\
USPC-ARK-4 & Mixed & 100 & 78 \\
USPC-ARK-5 & Pure & 100 & 114 \\
USPC-ARK-6 & Mixed & 100 & 86 \\
USPC-ARK-7 & Pure & 100 & 16 \\
USPC-ARK-8 & Pure & 100 & 454 \\
USPC-ARK-11 & Mixed & 20 & 14 \\
USPC-ARK-19 & Pure & 100 & 337 \\
USPC-ARK-20 & Mixed & 100 & 393 \\
USPC-ARK-21 & Pure & 100 & 573 \\
USPC-ARK-22 & Pure & 100 & 1222 \\
USPC-ARK-24 & Mixed & 54 & 60 \\
Ascites T0 & & & \\
USPC-ARK-19 & Pure & 100 & 1127 \\
USPC-ARK-22 & Pure & 100 & 827 \\
* & & & \\
MFI: Mean fluorescence intensity & & \\
& & &
\end{tabular}

ק.

